\title{
Glucosylceramide synthase regulates hepatocyte repair after concanavalin A-induced immune-mediated liver injury
}

\author{
Jian Gan $^{1}$, Qin Gao ${ }^{2}$, Li Li Wang ${ }^{3}$, Ai Ping Tian ${ }^{4}$, Long Dong Zhu ${ }^{4}$, Li Ting Zhang ${ }^{4}$, Wei Zhou ${ }^{5}$, Xiao Rong Mao \\ Corresp., 1, 4 , Jun Feng Li ${ }^{\text {Corresp. 1, 4, } 5}$ \\ 1 The First Clinical Medical College, Lanzhou University, Lanzhou, Gansu, China \\ 2 Physical Examination Center, The First Hospital of Lanzhou University, Lanzhou, Gansu, China \\ 3 Department of Radiology, The First Hospital of Lanzhou University, Lanzhou, Gansu, China \\ 4 Department of Infectious Diseases, The First Hospital of Lanzhou University, Lanzhou, Gansu, China \\ 5 Institute of Infectious Diseases, The First Hospital of Lanzhou University, Lanzhou, Gansu, China \\ Corresponding Authors: Xiao Rong Mao, Jun Feng Li \\ Email address: mxr2013@126.com, lijf12@Izu.edu.cn
}

Background: Sphingolipids produce pleiotropic signaling pathways, and participate in the pathological mechanism of hepatocyte apoptosis and necrosis during liver injury. However, the role of glucosylceramide synthase (GCS)--key enzyme that catalyzes the first glycosylation step, in liver injury is still vague.

Methods: All experiments were conducted using 7-9-week-old pathogen-free male C57BL/6 mice. Serum alanine aminotransferase (ALT) and aspartate aminotransferase (AST) levels were detected in murine models of liver disease, in addition to histological characterization of liver injuries. Quantitative real-time polymerase chain reaction ( $\mathrm{RRT}$-PCR) was used to detect the relative expression of the GCS, matrix metallopeptidase-1 (MMP-1), and tissue inhibitor of metalloproteinase-1 (TIMP-1) genes. The GCS was observed through a fluorescence microscope, and the flow cytometry was used to detect hepatocyte apoptosis. The concentrations of serum IL-4, IL-6, and IL-10 were measured using enzyme-linked immune-sorbent assay (ELISA) kit. MMP-1 and TIMP-1 protein expression was measured via western blot (WB) analysis.

Results: Con A is often used as a mitogen to activate T lymphocytes and promote mitosis. A single dose of Con A injected intravenously will cause a rapid increase of ALT and AST, which is accompanied by the release of cytokines that cause injury and necrosis of hepatocytes. In this study, we successfully induced acute immune hepatitis in mice by Con A. Con A administration resulted in GCS upregulation in liver tissues. Moreover, the mice in the Con A group had significantly higher levels of ALT, AST, IL-4, IL-6, IL-10 and increased hepatocyte apoptosis than the control group. In contrast, all of the aforementioned genes were significantly downregulated after the administration of a GCS siRNA or Genz-123346 (i.e., a glucosylceramide synthase inhibitor) to inhibit the GCS gene. Additionally, the histopathological changes observed herein were consistent with our ALT, AST, IL-4, IL-6, and IL-10 expression results. However, unlike this, hepatocyte apoptosis has been further increased on the basis of the Con A group. Moreover, our qRT-PCR and WB results indicated that the expression of MMP-1 in the Con A group was significantly lower than that in the control group, whereas TIMP-1 exhibited the opposite trend. Conversely, MMP-1 expression in the GCS siRNA and Genz-123346 groups was higher than that in the Con A group, whereas TIMP-1 expression was lower.

Conclusions: GCS inhibition reduces Con A-induced immune-mediated liver injury in mice, which may be 
due to the involvement of GCS in the hepatocyte repair process after injury. 


\section{Glucosylceramide synthase regulates hepatocyte}

2 repair after concanavalin A-induced immune-mediated

3 liver injury

4

5 Jian Gan ${ }^{1}$, Qin Gao ${ }^{2}$, Li Li Wang ${ }^{3}$, Ai Ping Tian ${ }^{4}$, Long Dong Zhu ${ }^{4}$, Li Ting Zhang ${ }^{4}$, Wei

6 Zhou ${ }^{5}$, Xiao Rong Mao ${ }^{1,4}$, Jun Feng Li ${ }^{1,4,5}$

7

$8{ }^{1}$ The First Clinical Medical College, Lanzhou University, Lanzhou, Gansu province, China

$9 \quad 2$ Physical Examination Center, The First Hospital of Lanzhou University, Lanzhou, Gansu

10 province, China

$11{ }^{3}$ Department of Radiology, The First Hospital of Lanzhou University, Lanzhou, Gansu province, 12 China

$13{ }^{4}$ Department of Infectious Diseases, The First Hospital of Lanzhou University, Lanzhou, Gansu 14 province, China

$15{ }^{5}$ Institute of Infectious Diseases, The First Hospital of Lanzhou University, Lanzhou, Gansu 16 province, China

Corresponding Author:

1. Jun Feng Li,

No. 1, Donggang West Road, Chengguan District, Lanzhou City, Gansu Province, 730000, China

22 Email address: junfenglee@126.com

2. Xiao Rong Mao

No. 1, Donggang West Road, Chengguan District, Lanzhou City, Gansu Province, 730000, China

Email address: mxr2013@126.com

27

\section{Abstract}

Background: Sphingolipids produce pleiotropic signaling pathways, and participate in the pathological mechanism of hepatocyte apoptosis and necrosis during liver injury. However, the role of glucosylceramide synthase (GCS)--key enzyme that catalyzes the first glycosylation step, in liver injury is still vague.

Methods: All experiments were conducted using 7-9-week-old pathogen-free male C57BL/6 mice. Serum alanine aminotransferase (ALT) and aspartate aminotransferase (AST) levels were detected in murine models of liver disease, in addition to histological characterization of liver injuries. Quantitative real-time polymerase chain reaction (qRT-PCR) was used to detect the 
37 relative expression of the GCS, matrix metallopeptidase-1 (MMP-1), and tissue inhibitor of

38

39

40

41

42

43

44

45

46

47

48

49

50

51

52

53

54

55

56

57

58

59

60

61

62

63

64

65

66

67

68

69

70

71

72

73

metalloproteinase-1 (TIMP-1) genes. The GCS was observed through a fluorescence microscope, and the flow cytometry was used to detect hepatocyte apoptosis. The concentrations of serum IL4, IL-6, and IL-10 were measured using enzyme-linked immune-sorbent assay (ELISA) kit. MMP-1 and TIMP-1 protein expression was measured via western blot (WB) analysis.

Results: Con A is often used as a mitogen to activate T lymphocytes and promote mitosis. A single dose of Con A injected intravenously will cause a rapid increase of ALT and AST, which is accompanied by the release of cytokines that cause injury and necrosis of hepatocytes. In this study, we successfully induced acute immune hepatitis in mice by Con A. Con A administration resulted in GCS upregulation in liver tissues. Moreover, the mice in the Con A group had significantly higher levels of ALT, AST, IL-4, IL-6, IL-10 and increased hepatocyte apoptosis than the control group. In contrast, all of the aforementioned genes were significantly downregulated after the administration of a GCS siRNA or Genz-123346 (i.e., a glucosylceramide synthase inhibitor) to inhibit the GCS gene. Additionally, the histopathological changes observed herein were consistent with our ALT, AST, IL-4, IL-6, and IL-10 expression results. However, unlike this, hepatocyte apoptosis has been further increased on the basis of the Con A group. Moreover, our qRT-PCR and WB results indicated that the expression of MMP-1 in the Con A group was significantly lower than that in the control group, whereas TIMP-1 exhibited the opposite trend. Conversely, MMP-1 expression in the GCS siRNA and Genz123346 groups was higher than that in the Con A group, whereas TIMP-1 expression was lower. Conclusions: GCS inhibition reduces Con A-induced immune-mediated liver injury in mice, which may be due to the involvement of GCS in the hepatocyte repair process after injury.

\section{Introduction}

MMP-1 and TIMP-1 are closely related to liver fibrosis, however, we do not know their role in early stage of liver fibrosis such as chronic hepatitis or even acute hepatitis. The purpose of this study was to illustrate the relationship between GCS and MMP-1 and TIMP-1, and the influence of MMP-1 on the outcome of acute immune hepatitis. Immune-related chronic liver injuries are among the key reasons for the occurrence and development of multiple liver diseases. For instance, autoimmune hepatitis is a progressive chronic inflammatory liver disease caused by abnormal autoimmune responses and is characterized by a loss of self-tolerance (You et al., 2021). Studies have shown that autoimmune hepatitis has a similar incidence worldwide, with an average of about 1.0 per 100000 . The prevalence depending on the geographical location, specifically, the range in North America is $22.8-42.9$ per 100 000, and the range in Europe is 10.7-19.44 per 100000 , which are generally higher than the 12.99 per 100000 in Asia (Delgado et al., 2013; Hurlburt et al., 2002; Lv et al., 2019). Long-term chronic inflammation and repeated liver injury gradually lead to liver fibrosis, cirrhosis, liver failure, and even death. Liver

Peer] reviewing PDF | (2021:04:60423:1:1:NEW 11 Jul 2021) 
74 tissue inflammation mediated by the liver's immune responses has been linked to the

75 pathological process of viral hepatitis, autoimmune liver disease, liver transplantation, alcoholic

76 liver disease, non-alcoholic fatty liver, and drug-induced liver injury, all of which lead to

77 hepatocyte injury, apoptosis, and necrosis (Heymann \& Tacke 2016; Zhang et al., 2019). Our

78 study found that sphingolipids can activate pleiotropic signaling pathways during immune-

79 mediated liver injury and contribute to hepatocyte injury, apoptosis, and necrosis (Brenner et al.,

80 2013). In fact, our previous studies (Li et al., 2014a; Li et al., 2014b; Li et al., 2017) and other

81 related studies (Grammatikos et al., 2015; Merrill et al., 2009) have demonstrated that

82 sphingolipids and their metabolic enzymes play a key role in the occurrence and development of

83 multiple liver diseases. Glycosphingolipids result from the glycosylation of sphingolipids, and

84 glucosylceramide (GC) is the central molecule in glycosphingolipid metabolism, which is

85 regulated by glucosylceramide synthase (GCS, EC: 2.4.1.80) (Liu et al., 2013). GCS, also

86 referred to as UDP-glucose ceramide glucosyltransferase (UGCG), is the key enzyme

87 that catalyzes the first glycosylation step during glycosphingolipid biosynthesis.

88 Therefore, GCS activity directly affects the balance between ceramide and GC in cells, which in

89 turn affects cell survival and immune function and may ultimately lead to disease onset. Based

90 on current studies, we hypothesized that GCS, the key enzyme in glycosphingolipid

91 metabolism, may affect the outcome of immune-mediated liver injury by regulating the

92 hepatocyte repair process after injury. Therefore, an in-depth analysis of the relationship

93 between GCS and immune-mediated liver injury would provide important insights into the

94 mechanisms that regulate the pathogenesis of many immune liver diseases.

95

96 Materials \& Methods

97 Ethics statement

98 This study was approved by the Institutional Animal Care and Use Committee and Scientific

99 Program of Lanzhou University (Ethical Application Ref: LDYYLL2018-100).

100 Experimental animals

101 Specific pathogen-free (SPF) male C57BL/6 mice were purchased from the Lanzhou University

102 animal laboratory (qualification certificate number: SCXK2018-0002). These mice were

103 congenic C57BL/6 (backcrossed for five generations) and were then inbred. All mice were

104 maintained in an SPF room (temperature $22-25{ }^{\circ} \mathrm{C}, 55 \%$ humidity, 12-hour light and dark cycle)

105 and fed standard laboratory chow and water ad libitum. All mice used in our experiments were

106 7-9-weeks-old and were similarly-sized ( $20 \pm 1 \mathrm{~g}$ body weight). In order to minimize potential

107 confounders, we chose mice born in the same litter, and the mice used in the experiment had

108 similar body weight.

109 Reagents 
110 The following reagents were used in our study: concanavalin A (Solarbio Life Science, Beijing);

111 4\% paraformaldehyde (Leagene Biotechnology, Beijing); Genz-123346 (MedChem Express,

112 USA); GCS siRNA: forward 5'-CCC GGU UAC ACC UCA ACA ATT-3'; reverse 5'-UUG

113 UUG AGG UGU AAC CGG GTT-3' (synthesized by GenePharma Co., Ltd. Shanghai);

114 Entranster ${ }^{\mathrm{TM}}$-in vivo (Engreen Biosystem, Beijing); RNAiso plus, Primescript RT reagent kit

115 with gDNA eraser, TB Green Premix Ex Taq II (Takara Biomedical Technology, Japan); primers

116 for GAPDH, GCS, MMP-1, and TIMP-1 (synthesized by Takara Biomedical Technology,

117 Japan); Mouse IL-4, IL-6, and IL-10 Elisa kits (Elabscience Biotechnology, Wuhan); RIPA lysis

118 solution, BCA Protein Assay Kit, polyvinylidene difluoride (PVDF) membranes, BeyoECL Plus

119 Kit and HRP-labeled secondary antibodies (Beyotime Biotechnology, Shanghai); fluorescent-

120 conjected secondary antibody (Jackson ImmunoResearch Inc., USA); Annexin V-FITC

121 apoptosis kit (BD Pharmingen, 556547, USA); anti-UGCG antibody (BOSTER Biological

122 Technology, BA2382, Wuhan); Cleaved caspase-3 antibody (Cell Signaling Technology, \#9664,

123 USA); anti-MMP-1 antibody (Biorbyt Ltd, orb214253, UK); anti-TIMP-1 antibody (Abcam,

124 ab61224, UK).

125 Experimental mouse models

126 The mice were allowed to acclimate for 3-7 days prior to the experiments. We selected a small

127 sample size because according to the results of our pre-experiment that 6 mice or even fewer

128 have shown significant statistical differences. Since the mice in the Con A-only group are at risk

129 of death, so the Con A-only groups originally numbered 7 mice. 31 mice were randomly divided

130 into five groups using a computer based random order generator, and the individual mouse was

131 considered the experimental unit within the study. The not treated (NT) group $(n=6)$ received a

132 single saline injection into the tail vein. The mice in the immune hepatitis group $(n=7)$ were

133 given a single intravenous injection of Con A ( $15 \mathrm{mg} / \mathrm{kg}$ body weight). The negative control

134 (NC) group $(\mathrm{n}=6)$ not treated with Con $\mathrm{A}$, but with GCS siRNA transfection complex

135 (Construction of transfection complex, siRNA $(\mu \mathrm{g})$ : Entranster ${ }^{\mathrm{TM}}$-in vivo $(\mu \mathrm{l})=2: 1$. Mix

136 thoroughly and incubate at room temperature for at least 15 minutes, then injected into mice via

137 tail vein). The fourth group ( $\mathrm{n}=6$ ) was treated with a single dose of GCS siRNA transfection

138 complex via tail vein injection two days before Con A administration. The last group $(n=6)$

139 received a GCS inhibitor (Genz-123346; $60 \mathrm{mg} / \mathrm{kg}$ ) twice a day via oral gavage two days before

140 Con A administration. After the Con A injection, the mice were kept in their cages for 24

141 additional hours. The animals were included in the study if they underwent successful

142 administration of Con A, GCS siRNA or Genz-123346, and the mice were excluded if died

143 prematurely. All experimental procedures were conducted thereafter. In this experiment, we

144 strictly controlled the use of experimental animals, so there were no extra surviving mice. And

145 there were no exclusions except the mice exhibited death, preventing the collection of

146 behavioral, blood and histological data. 
147 Liver enzyme analyses

14830 minutes before sacrifice, we administered $1 \%$ pentobarbital sodium $(80 \mathrm{mg} / \mathrm{kg}$, intraperitoneal 149 injection) to the mice. Finally, cervical dislocation was used for euthanizing mice. Blood was 150 obtained by removing the eyeball to analyze serum alanine aminotransferase (ALT) and aspartate aminotransferase (AST) as indicators of hepatocellular injury. Measurements of serum ALT

152

153

154

155

156

157

158

159

160

161

162

163

164

165

166

167

168

169

170

171

172

173

174

175

176

177

178

179

180

181

182

183

were made using an automatic biochemical analysis instrument (Olympus AU400 series, Tokyo, Japan).

\section{Histological and immunofluorescence}

Left outer lobe of liver was excised 24 hours after Con A administration, after which the liver samples were fixed in $4 \%$ paraformaldehyde and prepared for microscopic assessment using standard procedures. The samples were dehydrated, embedded in paraffin, and cut into 5- $\mu \mathrm{m}-$ thick sections along the long axis of the liver sample (Leica RM2235 Manual Rotary Microtome; Heidelberger, Germany), which were then stained with hematoxylin-eosin, and sections were blindly examined by a pathologist (Dr. Tian). As for immunofluorescence, after deparaffinization and antigen retrieval, $5 \%$ goat serum was added to the sections for blocking. The sections were then incubated with GCS-antibody $(1: 100)$ overnight at $4{ }^{\circ} \mathrm{C}$, followed by incubation with fluorescent-conjected secondary antibody $(1: 1000)$ protect from light for $1 \mathrm{~h}$ at $37^{\circ} \mathrm{C} .4,6$ diamino-2-phenylindole (DAPI) was used to stain the cell nuclei, and a fluorescence microscope (Nikon-ECLIPSE 80i, Tokyo, Japan) was used to capture the images. Images were acquired as 12-bit file format with both Nikon $4 \times$ and $40 \times$ objectives. Post imaging processing was performed using ImageJ 2x software (Rawak Software Inc., Stuttgart, Germany).

\section{RNA isolation and quantitative real-time PCR (qRT-PCR) analysis}

Total ribonucleic acid (RNA) was extracted from the liver using RNAiso Plus reagent, and RNA integrity was determined by agarose gel electrophoresis. 0.5 to $1.0 \mu \mathrm{g}$ of total extracted RNA was used to synthesize complementary DNA (cDNA) using Reverse Transcription Kit according to the manufacturer's instructions. qRT-PCR was performed on a Roche LightCycler480II (Roche Life Science, Basel, Switzerland) using 20- $\mu$ l reaction volumes (6.4 $\mu 1$ RNase-free water, $2 \mu \mathrm{l}$ cDNA, $0.8 \mu \mathrm{l}$ each of forward and reverse primers, and $10 \mu \mathrm{l}$ TB Green Premix Ex Taq II). qRT-PCR analyses were conducted using the following primers: GAPDH 5'-TGT GTC CGT CGT GGA TCT GA-3' (forward), 5'-TTG CTG TTG AAG TCG CAG GAG-3' (reverse); GCS 5'-TTC GAG GGC GTG TTA TCC ATC-3' (forward), 5'-CAA ATG GGC TGG CTC AGT AAG A-3' (reverse); MMP-1 5'-TCC CTG GAA TTG GCA ACA AAG-3' (forward), 5'-GCA TGA CTC TCA CAA TGC GAT TAC-3' (reverse); TIMP-1 5'-GGA ACG GAA ATT TGC ACA TCA G-3' (forward), 5'-CTG ATC CGT CCA CAA ACA GTG AG-3' (reverse). Relative gene expression was calculated using the comparative cycle threshold method using GAPDH as reference.

Flow cytometry of apoptosis 
184 In short, the liver was excised and digested by trypsin. Obtain primary hepatocytes and filter 185 repeatedly through cell sieves. The cells were harvested and incubated with reagents from the 186 Annexin V-FITC apoptosis kit according to the manufacturer's instructions, and the cells were 187 counted using a flow cytometer (Beckman CytoFLEX, California, USA).

\section{Measurement of cytokines in the serum}

189 Serum was collected from individual mice, after which commercially available ELISA kits were 190 used to determine serum IL-4, IL-6, and IL-10 using a multifunctional microplate reader 191 (Thermo Scientific, Massachusetts, USA) according to the manufacturer's instructions. The 192 mouse IL-4, IL-6, and IL-10 specific polyclonal antibodies were pre-coated onto 96-well plates. 193 The standards, test samples, and biotinylated detection antibodies were then pipetted into the 194 wells, after which unbound biotinylated antibodies were washed away using washing buffer. 195 Biotin-conjugated HRP was pipetted into each microplate well and incubated, then the wells 196 were washed and substrate stop solution was added. Only the wells that contained the 197 corresponding cytokines and biotin-conjugated antibody exhibited a color shift from blue to 198 yellow, after which the optical absorbance at $450 \mathrm{~nm}$ was determined using a microplate 199 absorbance reader (Thermo Scientific Varioskan Flash, Massachusetts, USA).

200 Western blot analysis

201 Liver tissues were homogenized in ice-cold lysis buffer and incubated for 10 minutes, then 202 centrifuged (Beckman Coulter life sciences, California, USA) at 12,000 $\times \mathrm{g}$ for 10 minutes at 4 $203{ }^{\circ} \mathrm{C}$. Protein concentrations were quantified using the BCA Protein Assay Kit. A loading buffer 204 was then added and the proteins were boiled for 5-10 minutes. Equal amounts of whole protein $205(30 \mu \mathrm{g})$ were separated on 12\% acrylamide gels using sodium dodecyl sulfate-polyacrylamide 206 gel electrophoresis. The proteins were then transferred from the gel to PVDF membranes and 207 blocked in TBST containing 5\% non-fat milk for 1 hour. Next, the membranes were incubated 208 overnight at $4{ }^{\circ} \mathrm{C}$ with a specific primary antibody according to the recommended dilution ratio 209 in the instructions. After the incubation period, each PVDF membrane was washed 3-5 times (8 210 minutes per wash) with TBST and incubated for 1.5 hours at room temperature with HRP211 labeled secondary antibodies. The bound antibodies were then visualized using enhanced

212 chemiluminescence detection reagents and exposed using an Amersham Imager 600 system (GE 213 Healthcare, Boston, USA). The signals were analyzed and quantified using the ImageJ 2x 214 software.

215 Statistical analyses

216 All data were processed using SPSS 16.0 for Windows software (SPSS, Inc., Chicago, IL), and 217 the results were reported as mean \pm standard deviation (SD). The Student $t$ test was used to 218 compare the experimental groups. 95\% confidence interval for each statistical analysis and a $P$ 219 value $<0.05$ was considered statistically significant. 


\section{Results}

\section{General conditions of mice after Con A administration}

223 Prior to the administration of Con A, no significant differences in mental state, behavior, or 224 appearance were observed among the mice regardless of the experimental group. However, the 225 mice treated with Con A alone exhibited malaise, reduced activity, trembling, and huddling to 226 remain warm. Moreover, they responded poorly to external stimuli and their fur became fluffy 227 and messy. The other groups did not exhibit these traits. Moreover, among all of the tested 228 groups, only the Con A-only group exhibited one death. Due to overt behavioral activity the 229 experimenter could not be blinded to whether the animal was injected with Con A.

\section{GCS inhibition reduces Con A-induced hepatic enzyme upregulation}

As shown in Fig. 1A and 1B, serum ALT and AST levels were significantly elevated in the Con A group compared to those in the NT group. Moreover, we noticed that the GCS mRNA expression of the Con A-exposed mice was significantly higher than that of the NT group (Fig. 1C). We then treated the mice with GCS siRNA and Genz-123346 to suppress GCS expression. As expected, serum ALT and AST levels were significantly lower in the mice that were pretreated with GCS siRNA and Genz-123346 than in the mice that were exposed to Con A alone. GCS inhibition ameliorates Con A-induced liver injury

238

239

240

241

242

243

244

245

246

247

248

249

250

251

252

253

254

255

256

257

Based on our histological analyses of liver samples, the mice in the Con A group (Fig. 2B) exhibited areas with widespread necrosis and extensive infiltration of inflammatory cells around the central veins (i.e., clear signs of inflammation) compared to the NT group (Fig. 2A). In contrast, the liver tissue sections from the mice that were pre-treated with GCS siRNA (Fig. 2C) and Genz-123346 (Fig. 2D) only exhibited some isolated areas of necrotic tissue, indicating that the majority of the hepatocytes were less sensitive to Con A-induced liver injury. By immunofluorescence (Fig. 3), we observed the GCS level was increased in the Con A group. As expected, the GCS siRNA+Con A and Genz+Con A groups showed a decrease GCS expression compared to the Con A group.

\section{Increased hepatocytes apoptosis rate after inhibiting GCS}

Flow cytometry analysis (Fig. 4A and 4B) revealed that the GCS siRNA+Con A and Genz+Con A group showed more apoptosis rate compared to the Con A group, especially the Genz+Con A group, with an apoptosis rate of $21.6 \%$ compared with the other three groups $1.36 \%, 7.05 \%$, $12.5 \%$, respectively. Similarly, inhibition of GCS in normal mice (NC group) caused hepatocyte apoptosis, but the apoptosis rate was not as obvious as that in Con A-treated mice.

\section{Inhibiting GCS alleviates the release of inflammatory cytokines caused by Con A-induced} liver immune responses

Con A-induced liver injuries provide a robust basis for the establishment of immune liver injury models, as this compound can activate $\mathrm{T}$ lymphocytes and promotes the release of large amounts of cytokines into the bloodstream. Next, to investigate the role of 
258 GCS in Con A-induced autoimmune hepatitis, we quantified the levels of multiple cytokines in 259 the serum of mice. Con A-injected mice exhibited significantly higher IL-4, IL-6, and 260 IL-10 serum levels (Fig. 5A, 5B, and 5C, respectively) compared to the NT group. As 261 expected, after GCS suppression, the mice in the GCS siRNA+Con A and Genz+Con A 262 groups had significantly lower IL-4, IL-6, and IL-10 serum levels compared to the Con 263 A group.

264 GCS affects the hepatocyte repair process by regulating MMP-1 and TIMP-1

265 Next, the levels of MMP-1 (Fig. 6A and 6D) and TIMP-1 (Fig. 6B and 6E) were quantified to 266 explore whether the mitigation of immune-mediated liver injury by GCS was due to an enhanced hepatocyte repair function. Compared with the NT group, the TIMP-1 levels in the liver were significantly higher after con A injection, whereas MMP-1 exhibited the opposite trend.

270 Conversely, hepatic MMP-1 levels increased compared with the Con A group, whereas TIMP-1

271 decreased gradually. However, inhibition of GCS in normal mice (NC group) have no obvious

272

\section{Discussion}

274 Increasing evidence suggests a link between GCS expression and disease onset, thus 275 highlighting the value of GCS as a promising therapeutic target (Pavlova et al., 2015; 276 Wang et al., 2015; Wegner et al., 2018). Here, we demonstrated that the GCS played a 277 key role in immune hepatitis. GCS inhibition had beneficial effects in murine models of 278 Con A-induced hepatitis, and these reductions in liver injuries appeared to be related to 279 the regulation of hepatocyte repair function by GCS.

280 The liver is not only a major metabolic organ but also an important immune system 281 organ. Moreover, its various constituent cells [e.g., T lymphocytes, B lymphocytes, natural killer (NK) cells, and natural killer T (NKT) cells] are involved in several immune responses (Gan et al., 2020; Zhang et al., 2019). Physical and chemical factors, viruses, drugs, and alcohol can lead to chronic hepatocyte injury, apoptosis, and necrosis via the activation of immune responses, thus resulting in liver diseases such as viral hepatitis, druginduced liver injury, autoimmune liver disease, and alcoholic liver disease (Smyk et al., 2018; Zhang et al., 2019). Therefore, immune-related chronic liver injury has recently been linked to the occurrence and development of multiple liver diseases. T lymphocytemediated cellular immune responses are generally known to be the main factor that leads to hepatocyte injury in immune hepatitis (Loggi et al., 2014). This is especially true for natural killer T cells, which are abundant in the liver, particularly in rodents (Opasawatchai \& Matangkasombut 2015). NKT cells secrete high levels of cytokines 294 (including Th1 and Th2) when stimulated by antigenic substances, thereby participating in the regulation of innate and adaptive immunity and affecting the outcome of immune- 
295

296

297

298

299

300

301

302

303

304

305

306

307

308

309

310

311

312

313

314

315

316

317

318

319

320

321

322

323

324

325

326

327

328

329

330

331

mediated liver injury (Popovic et al., 2017). Recent studies have demonstrated that sphingolipids play a key role in regulating the development, maturation, and homeostasis of NKT cells in the liver. Therefore, mice lacking enzymes for sphingolipid synthesis exhibit reduced immunity and are susceptible to pathogen infection (Saroha et al., 2017). GCS is a key enzyme for the synthesis of glycosylated sphingolipids, as this enzyme can generate all complex glycosyl phospholipids precursors, including glucosylceramide (GC). GCS is a key node for lipid glycosylation and non-glycosylation regulation, and thus its activity directly affects the metabolic balance of glycosylated and non-glycosylated sphingolipids, which in turn affects cell survival and immune function (Popovic et al., 2017). Therefore, GCS plays a key role in immune hepatitis and affects its outcome. Con A is a kind of plant lectin extracted from jack beans (Canavalia ensiformis). This compound binds to the sugar chain residues on the cell surface to promote cell aggregation and $\mathrm{T}$ lymphocyte mitosis, thus inducing lymphocyte and macrophage toxicity (Wang et al., 2017). Therefore, Con A-induced liver injury is typically used as a model to simulate human immune hepatitis (Li et al., 2015). In this study, we constructed a liver injury model by injecting Con A $(15 \mathrm{mg} / \mathrm{kg})$ into the tail vein of mice, after which the serum levels of ALT and AST increased rapidly. ALT is ubiquitous in the body; however, its highest concentrations are typically observed in the cytoplasm and mitochondria of hepatocytes, which makes this enzyme a good indicator for hepatocyte injury diagnosis (Fullerton et al., 2013; Tsutsui \& Nishiguchi 2014). Moreover, Con A-treated mice also released a large amount of inflammatory cytokines (IL-4, IL-6, and IL-10) into their bloodstream, and histopathological examination revealed observable liver injuries and necrosis. In short, all of the aforementioned reports are consistent with the symptoms of immune hepatitis, including inflammatory cell infiltration, hepatocyte necrosis, and the release of a large number of inflammatory factors (Wang et al., 2015). Genz-123346 is a selective inhibitor of GCS, which specifically inhibit GCS, whether in vivo (Natoli et al., 2010) or in vitro (Chai et al., 2011). The use of Genz-123346 will significantly inhibit the activity of GCS and reduce the level of GC, which leads to an increase in the level of ceramide (the precursor of glucosylceramide), but it didn't significantly alter the levels of other sphingolipids such as sphingosine 1-phosphate, sphingomyelin or sphingosine (Koike et al., 2019). Afterward, we inhibited the activity of GCS by administering effective doses of GCS siRNA and Genz-123346 (i.e., a GCS chemical inhibitor). As expected, the levels of serum ALT, AST, and inflammatory cytokines (IL-4, IL-6, and IL-10) in mice were significantly reduced, and liver histopathological analyses revealed a substantial reduction in liver injuries. Interestingly, as GCS is inhibited, the rate of hepatocyte apoptosis is increasing. This may be that after GCS is inhibited, the conversion of ceramide into GC is reduced, and a large amount of ceramide accumulates in hepatocyte and causing apoptosis (Stefanovic et al., 2016). In addition, GCS may be the necessary protein for the proliferation, since the GCS knockout mice exhibit embryonic lethality due to massive apoptosis (Yamashita et

PeerJ reviewing PDF | (2021:04:60423:1:1:NEW 11 Jul 2021) 
332 al., 1999). Furthermore, this may be a self-protection mechanism, because the liver resists severe 333 injury by increasing the number of apoptosis. These results suggest that GCS inhibition can 334 reduce Con A-induced liver injury in mice and inhibits the release of inflammatory cytokines.

335 MMP-1 is mainly produced by hepatic stellate cells and is a type of $\mathrm{Zn}^{2+}$ - and $\mathrm{Ca}^{2+}$-dependent endogenous protease that exists in zymogen form. It can degrade a variety of extracellular matrix (ECM) components to reduce collagen deposition, and play a critical role in alleviating liver fibrosis. Excessive ECM deposition is known to cause organ fibrosis (e.g., liver fibrosis) and therefore the relationship between MMP-1 and liver fibrosis has recently garnered increasing attention from the scientific community. Liver fibrosis is a common terminal manifestation of chronic liver disease characterized by persistent liver parenchymal injury, activation and recruitment of immune cells, activation of HSC, and excessive secretion of ECM, all of which lead to scar formation (Roderfeld 2018). Therefore, the liver appears to undergo a long inflammatory response period before the development of liver fibrosis, during which inflammatory injuries are repeatedly repaired. If this inflammatory response is not effectively controlled, chronic liver disease ultimately develops into liver fibrosis. Previous studies have demonstrated that MMP-1 is not only an important indicator of ECM degradation during liver fibrosis, but also maintains the integrity of the cell structure and the basement membrane of the liver sinus during the repair process after injury, which occurs prior to the development of liver fibrosis (Roderfeld 2018). Therefore, MMP-1 may play a key role in hepatitis onset and can thus be used as an indicator of cell repair capacity. Tissue inhibitor of metalloproteinase-1 (TIMP-1) has a specific inhibitory effect on MMP-1, which reduces the degradation of ECM by MMP-1 and leads to the development of liver fibrosis. Therefore, it is believed that the dynamic balance between MMP-1 and TIMP-1 plays a decisive role in the outcome of liver injury. In order to study how GCS can reduce hepatocyte injury in Con A-induced immune hepatitis, we chose to explore MMP-1 and TIMP-1. In this study, the experimental mice exhibited obvious signs of liver injury with a significant increase in inflammatory cytokines after Con A administration, all of which coincided with a substantial upregulation of the GCS gene. Furthermore, both our qRTPCR and WB results supported the decrease in MMP-1 and increase in TIMP-1 expression in the livers of the Con A group mice. Interestingly, the opposite occurred after GCS inhibition, resulting in a marked decrease in liver injuries. During liver injury, the upregulation of TIMP-1 inhibits MMP-1 activity, resulting in a decrease in the repair capacity of hepatocytes, and therefore the integrity of the hepatocyte structure and the normal morphology of the basement membrane of the liver sinus cannot be maintained. Liver injuries then become progressively more severe and gradually develop into liver fibrosis (Bataller \& Brenner 2005). Conversely, a decrease in TIMP-1 leads to a weakened inhibition of MMP-1, which protects the hepatocytes. 368 Here, we provide further evidence that inhibiting GCS can increase MMP-1 expression, thereby enhancing hepatocyte repair. 
369 There are several potential limitations of this study that need to be discussed. First, although our 370 preliminary findings suggest that GCS affects the outcome of immune liver injury by regulating 371 the levels of MMP-1 and TIMP-1 in liver tissue, we do not know whether GCS regulates other

372 types of MMPs and TIMPs, and future studies should also determine whether there are

373 differences between MMP and TIMP subtype functions and if there are synergistic or

374 antagonistic interactions between them. In addition, this study is not thorough enough on the 375 changes in $\mathrm{T}$ lymphocytes and their subtypes in Con A induced immune hepatitis. This will be

376

377

378

379

380

381

382

383

384

385

386

387

388

389

390

391

392

393

394

395

396

397

398

399

400

401

402

403

404

405 the focus of our future work, and we are already working on it.

\section{Conclusions}

Con A-induced organ injury provides a simple means to recreate liver-specific injuries and does not require pre-sensitization (Heymann et al., 2015). Using this approach to create a murine model of immune-mediated liver injury, our study demonstrated the critical role of GCS in liver disease onset. GCS inhibition enhances the self-repair capacity of hepatocytes, thus alleviating disease symptoms. Moreover, our findings suggest that regulating MMP-1 through different means may serve as a promising treatment for liver injury; however, these findings must be further confirmed in other models.

\section{Acknowledgements}

We are grateful to the Central Laboratory of the First Hospital of Lanzhou University for providing us with experimental support. In addition, we are grateful to all the staff in this study.

\section{Data availability}

Since our paper has not been published so cannot be made freely available, but available from the corresponding author on reasonable request. To maximize the objectivity of the presented analyses, we preregistered (https://isisn.nsfc.gov.cn/egrantweb/) this study with its two hypotheses, its planned methods, and its complete plan of data analysis before the start of data collection, and we closely adhered to our plan.

\section{References}

Bataller R, and Brenner DA. 2005. Liver fibrosis. Journal of Clinical Investigation 115:209218. DOI 10.1172/JCI24282.

Brenner C, Galluzzi L, Kepp O, and Kroemer G. 2013. Decoding cell death signals in liver inflammation. J. Hepatol 59:583-594. DOI 10.1016/j.jhep.2013.03.033.

Chai L, McLaren RP, Byrne A, Chuang WL, Huang Y, Dufault MR, Pacheco J, Madhiwalla S, Zhang X, Zhang M, Teicher BA, Carter K, Cheng SH, Leonard JP, Xiang Y, Vasconcelles M, Goldberg MA, Copeland DP, Klinger KW, Lillie J, 
406

407

408

409

410

411

412

413

414

415

416

417

418

419

420

421

422

423

424

425

426

427

428

429

430

431

432

433

434

435

436

437

438

439

440

441

442

Madden SL, and Jiang YA. 2011. The chemosensitizing activity of inhibitors of glucosylceramide synthase is mediated primarily through modulation of P-gp function. International Journal of Oncology 38:701-711. DOI 10.3892/ijo.2010.888.

Delgado JS, Vodonos A, Malnick S, Kriger O, Wilkof-Segev R, Delgado B, Novack V, Rosenthal A, Menachem Y, Melzer E, and Fich A. 2013. Autoimmune hepatitis in southern Israel: a 15-year multicenter study. J. Dig. Dis. 14:611-618. DOI 10.1111/17512980.12085.

Fullerton AM, Roth RA, and Ganey PE. 2013. Pretreatment with TCDD exacerbates liver injury from Concanavalin A: critical role for NK cells. Toxicological Sciences 136:72-85. DOI 10.1093/toxsci/kft174.

Gan J, Zheng SJ, Mao XR, and Li JF. 2020. The role of glucosylceramide and glucosylceramide synthase in liver disease: from bench to bedside - Review. Acta Biochimica Polonica. DOI 10.18388/abp.2020_5478.

Grammatikos G, Ferreiros N, Bon D, Schwalm S, Dietz J, Berkowski C, Fitting D, Herrmann E, Zeuzem S, Sarrazin C, and Pfeilschifter J. 2015. Variations in serum sphingolipid levels associate with liver fibrosis progression and poor treatment outcome in hepatitis C virus but not hepatitis B virus infection. Hepatology 61:812-822. DOI 10.1002/hep.27587.

Heymann F, Hamesch K, Weiskirchen R, and Tacke F. 2015. The concanavalin A model of acute hepatitis in mice. Lab. Anim. 49:12-20. DOI 10.1177/0023677215572841.

Heymann F, and Tacke F. 2016. Immunology in the liver--from homeostasis to disease. Nat Rev Gastroenterol Hepatol 13:88-110. DOI 10.1038/nrgastro.2015.200.

Hurlburt KJ, McMahon BJ, Deubner H, Hsu-Trawinski B, Williams JL, and Kowdley KV. 2002. Prevalence of autoimmune liver disease in Alaska Natives. Am. J. Gastroenterol. 97:2402-2407. DOI 10.1111/j.1572-0241.2002.06019.x.

Koike K, Berdyshev EV, Mikosz AM, Bronova IA, Bronoff AS, Jung JP, Beatman EL, Ni K, Cao D, Scruggs AK, Serban KA, and Petrache I. 2019. Role of Glucosylceramide in Lung Endothelial Cell Fate and Emphysema. American Journal of Respiratory and Critical Care Medicine 200:1113-1125. DOI 10.1164/rccm.201812-2311OC.

Li J, Xia Y, Liu T, Wang J, Dai W, Wang F, Zheng Y, Chen K, Li S, Abudumijiti H, Zhou Z, Wang J, Lu W, Zhu R, Yang J, Zhang H, Yin Q, Wang C, Zhou Y, Lu J, Zhou Y, and Guo C. 2015. Protective effects of astaxanthin on ConA-induced autoimmune hepatitis by the JNK/p-JNK pathway-mediated inhibition of autophagy and apoptosis. PLoS One 10:e0120440. DOI 10.1371/journal.pone.0120440.

Li JF, Qu F, Zheng SJ, Ren JY, Wu HL, Liu M, Liu H, Ren F, Chen Y, Zhang JL, and Duan ZP. 2014a. Plasma sphingolipids as potential indicators of hepatic necroinflammation in patients with chronic hepatitis $\mathrm{C}$ and normal alanine 
443

444

445

446

447

448

449

450

451

452

453

454

455

456

457

458

459

460

461

462

463

464

465

466

467

468

469

470

471

472

473

474

475

476

477

478

479

aminotransferase level. PLoS One 9:e95095. DOI 10.1371/journal.pone.0095095.

Li JF, Qu F, Zheng SJ, Wu HL, Liu M, Liu S, Ren Y, Ren F, Chen Y, Duan ZP, and Zhang JL. 2014b. Elevated plasma sphingomyelin (d18:1/22:0) is closely related to hepatic steatosis in patients with chronic hepatitis C virus infection. European Journal of Clinical Microbiology \& Infectious Diseases 33:1725-1732. DOI 10.1007/s10096-014-2123-X.

Li JF, Zheng SJ, Wang LL, Liu S, Ren F, Chen Y, Bai L, Liu M, and Duan ZP. 2017. Glucosylceramide synthase regulates the proliferation and apoptosis of liver cells in vitro by Bcl2/Bax pathway. Mol. Med. Rep. 16:7355-7360. DOI 10.3892/mmr.2017.7580.

Liu YY, Hill RA, and Li YT. 2013. Ceramide glycosylation catalyzed by glucosylceramide synthase and cancer drug resistance. Advances in Cancer Research 117:59-89. DOI 10.1016/B978-0-12-394274-6.00003-0.

Loggi E, Gamal N, Bihl F, Bernardi M, and Andreone P. 2014. Adaptive response in hepatitis B virus infection. J. Viral. Hepat. 21:305-313. DOI 10.1111/jvh.12255.

Lv T, Li M, Zeng N, Zhang J, Li S, Chen S, Zhang C, Shan S, Duan W, Wang Q, Wu S, You H, Ou X, Ma H, Zhang D, Kong Y, and Jia J. 2019. Systematic review and metaanalysis on the incidence and prevalence of autoimmune hepatitis in Asian, European, and American population. J. Gastroenterol. Hepatol. 34:1676-1684. DOI 10.1111/jgh.14746.

Merrill AH, Jr., Stokes TH, Momin A, Park H, Portz BJ, Kelly S, Wang E, Sullards MC, and Wang MD. 2009. Sphingolipidomics: a valuable tool for understanding the roles of sphingolipids in biology and disease. Journal of Lipid Research 50 Suppl:S97-102. DOI 10.1194/j1r.R800073-JLR200.

Natoli TA, Smith LA, Rogers KA, Wang B, Komarnitsky S, Budman Y, Belenky A, Bukanov NO, Dackowski WR, Husson H, Russo RJ, Shayman JA, Ledbetter SR, Leonard JP, and Ibraghimov-Beskrovnaya O. 2010. Inhibition of glucosylceramide accumulation results in effective blockade of polycystic kidney disease in mouse models. Nature Medicine 16:788-792. DOI 10.1038/nm.2171.

Opasawatchai A, and Matangkasombut P. 2015. iNKT cells and their potential lipid ligands during viral infection. Frontiers in Immunology 6. ARTN 378. DOI 10.3389/fimmu.2015.00378.

Pavlova EV, Archer J, Wang S, Dekker N, Aerts JM, Karlsson S, and Cox TM. 2015. Inhibition of UDP-glucosylceramide synthase in mice prevents Gaucher diseaseassociated B-cell malignancy. Journal of Pathology 235:113-124. DOI $10.1002 /$ path. 4452 .

Popovic ZV, Rabionet M, Jennemann R, Krunic D, Sandhoff R, Grone HJ, and Porubsky S. 2017. Glucosylceramide Synthase Is Involved in Development of Invariant Natural Killer T Cells. Front Immunol 8:848. DOI 10.3389/fimmu.2017.00848. 
480

481

482

483

484

485

486

487

488

489

490

491

492

493

494

495

496

497

498

499

500

501

502

503

504

505

506

507

508

509

510

511

512

513

514

515

516

Roderfeld M. 2018. Matrix metalloproteinase functions in hepatic injury and fibrosis. Matrix Biol. 68-69:452-462. DOI 10.1016/j.matbio.2017.11.011.

Saroha A, Pewzner-Jung Y, Ferreira NS, Sharma P, Jouan Y, Kelly SL, Feldmesser E, Merrill AH, Jr., Trottein F, Paget C, Lang KS, and Futerman AH. 2017. Critical Role for Very-Long Chain Sphingolipids in Invariant Natural Killer T Cell Development and Homeostasis. Front Immunol 8:1386. DOI 10.3389/fimmu.2017.01386.

Smyk DS, Mavropoulos A, Mieli-Vergani G, Vergani D, Lenzi M, and Bogdanos DP. 2018. The Role of Invariant NKT in Autoimmune Liver Disease: Can Vitamin D Act as an Immunomodulator? Can. J. Gastroenterol. Hepatol. 2018:8197937. DOI 10.1155/2018/8197937.

Stefanovic M, Tutusaus A, Martinez-Nieto GA, Barcena C, de Gregorio E, Moutinho C, Barbero-Camps E, Villanueva A, Colell A, Mari M, Garcia-Ruiz C, FernandezCheca JC, and Morales A. 2016. Targeting glucosylceramide synthase upregulation reverts sorafenib resistance in experimental hepatocellular carcinoma. Oncotarget 7:8253-8267. DOI 10.18632/oncotarget.6982.

Tsutsui H, and Nishiguchi S. 2014. Importance of Kupffer cells in the development of acute liver injuries in mice. Int J Mol Sci 15:7711-7730. DOI 10.3390/ijms15057711.

Wang L, Zhang W, Ge CH, Yin RH, Xiao Y, Zhan YQ, Yu M, Li CY, Ge ZQ, and Yang XM. 2017. Toll-like receptor 5 signaling restrains T-cell/natural killer T-cell activation and protects against concanavalin A-induced hepatic injury. Hepatology 65:2059-2073. DOI 10.1002/hep.29140.

Wang T, Wei J, Wang N, Ma JL, and Hui PP. 2015. The glucosylceramide synthase inhibitor PDMP sensitizes pancreatic cancer cells to MEK/ERK inhibitor AZD-6244. Biochemical and Biophysical Research Communications 456:821-826. DOI 10.1016/j.bbrc.2014.12.019.

Wegner MS, Gruber L, Mattjus P, Geisslinger G, and Grosch S. 2018. The UDP-glucose ceramide glycosyltransferase (UGCG) and the link to multidrug resistance protein 1 (MDR1). BMC Cancer 18:153. DOI 10.1186/s12885-018-4084-4.

Yamashita T, Wada R, Sasaki T, Deng C, Bierfreund U, Sandhoff K, and Proia RL. 1999. A vital role for glycosphingolipid synthesis during development and differentiation. Proc. Natl. Acad. Sci. U S A 96:9142-9147. DOI 10.1073/pnas.96.16.9142.

You Z, Li Y, Wang Q, Zhao Z, Li Y, Qian Q, Li B, Zhang J, Huang B, Liang J, Chen R, Lyu Z, Chen Y, Lian M, Xiao X, Miao Q, Fang J, Lian Z, Gershwin ME, Tang R, and Ma X. 2021. The Clinical Significance of Hepatic CD69(+) CD103(+) CD8(+) Resident Memory T Cells in Autoimmune Hepatitis. Hepatology. DOI 10.1002/hep.31739.

Zhang T, Hu J, Wang X, Zhao X, Li Z, Niu J, Steer CJ, Zheng G, and Song G. 2019. 
MicroRNA-378 promotes hepatic inflammation and fibrosis via modulation of the NFkappaB-TNFalpha pathway. $J$ Hepatol 70:87-96. DOI 10.1016/j.jhep.2018.08.026. 
Figure 1

Serum ALT, AST levels and relative expression of GCS mRNA.

(A, B) Serum ALT and AST levels. (C) The relative expression of GCS mRNA. (D) $1.2 \%$ Tris, borate and ethylenediamine tetraacetic acid (TBE) agarose gel electrophoresis of representative RNA samples.

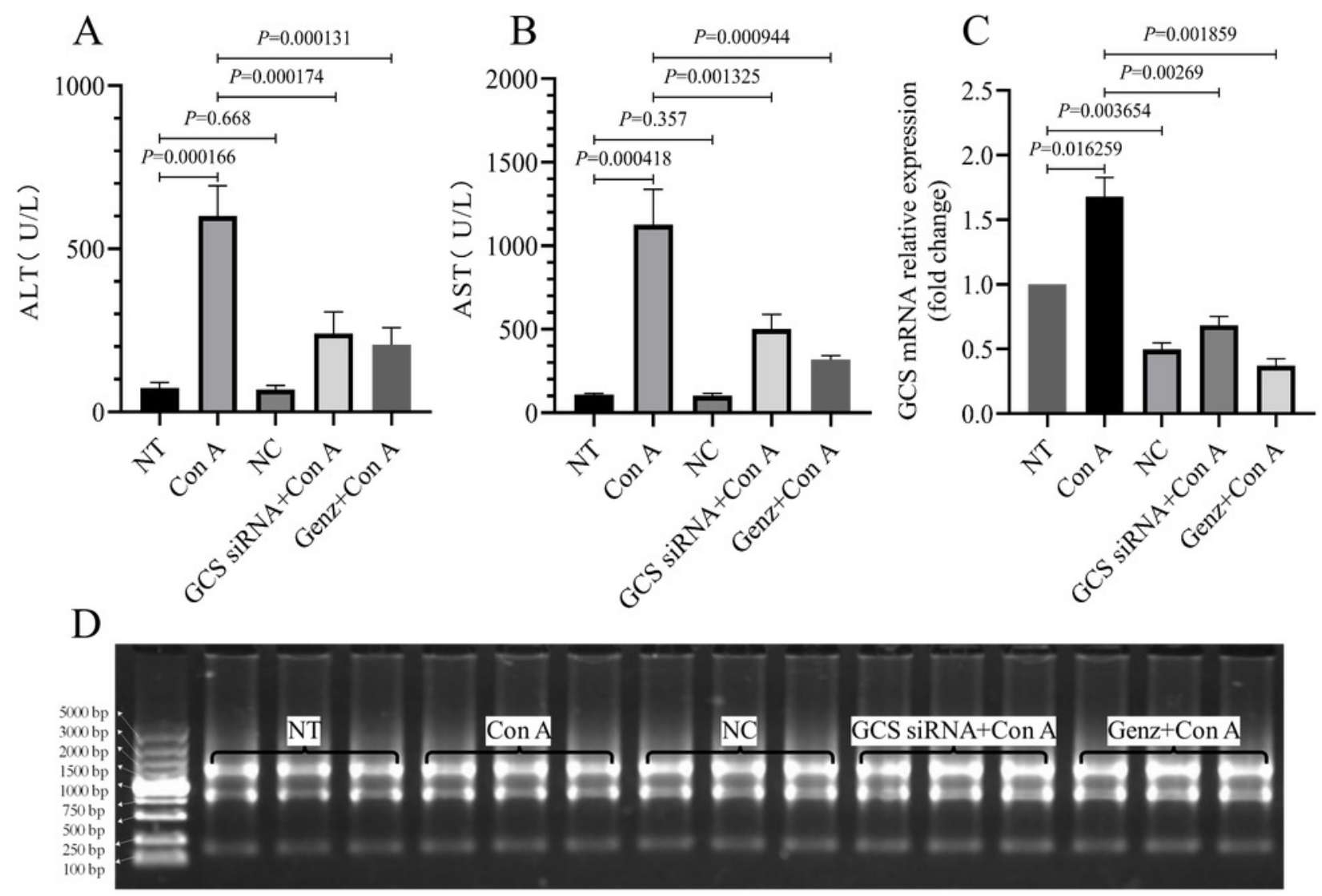




\section{Figure 2}

Photomicrographs of representative sections of liver at 24 hours after Con A injection.

( $A$ and F) Normal liver section, no necrotic areas. (B and G) Widespread necrotic areas and inflammatory cells infiltrate, mainly lymphocytes. ( $C$ and $H$ ) no necrotic areas. ( $D$ and I) Some isolated necrotic areas and lymphocytes infiltrate. (E and J) Some isolated necrotic areas and lymphocytes infiltrate (Note: A-E represents the NT group, Con A group, NC group, GCS siRNA+Con A group and Genz+Con A group in turn, $H \& E, 4 \times$. F-J represents the NT group, Con A group, NC group, GCS siRNA+Con A group and Genz+Con A group in turn, H\&E, 40X. Red wireframe represents the necrotic areas, and black arrows indicate the central vein of hepatic lobules). 


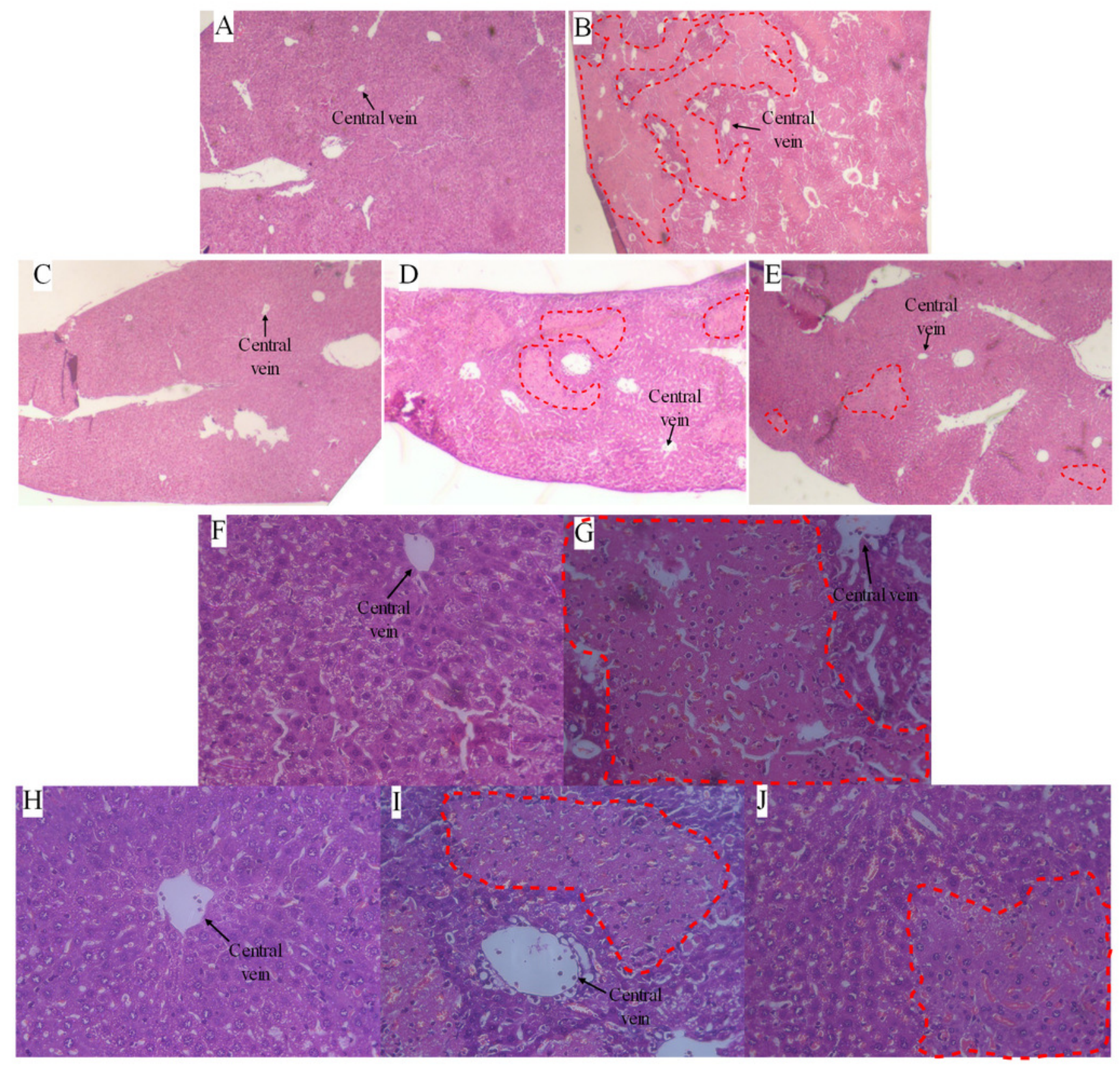


Figure 3

Expression of GCS protein in liver.

(A) The representative immunofluorescent images of GCS. DAPI labeled nucleus (blue);

DyLight 594 (red) labeled GCS. Scale bars are $50 \mu \mathrm{m}$. (B) GCS protein were analyzed by western blot. (C) Relative levels of GCS protein corrected by GAPDH. 


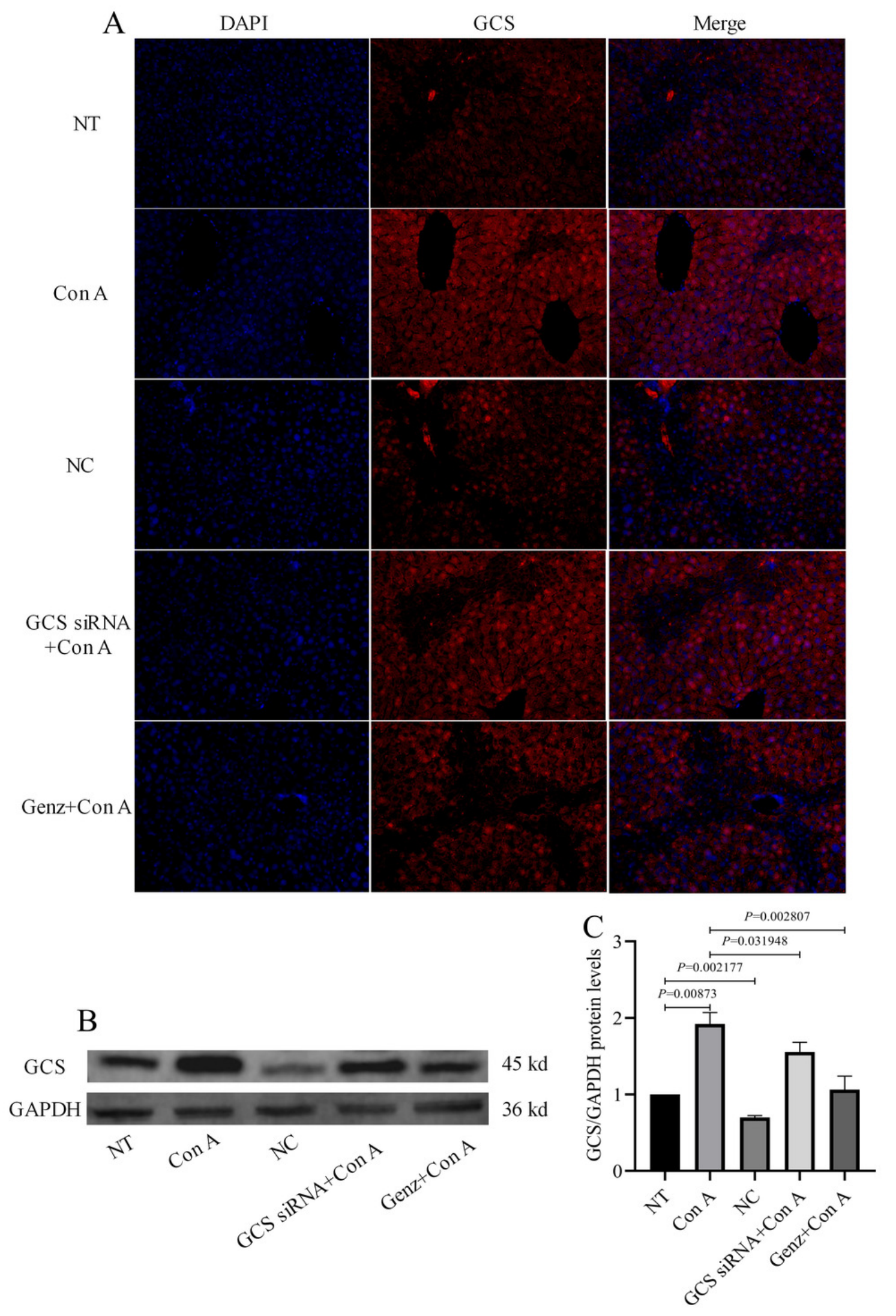


Figure 4

Apoptosis rate of hepatocyte.

(A) Flow cytometric analysis of cellular apoptosis using annexin V/PI double staining. (B)

Statistical analysis of hepatocyte apoptosis rate. (C) Cleaved caspase-3 activity were analyzed by western blot. (D) Relative levels of cleaved caspase-3 activity corrected by GAPDH.

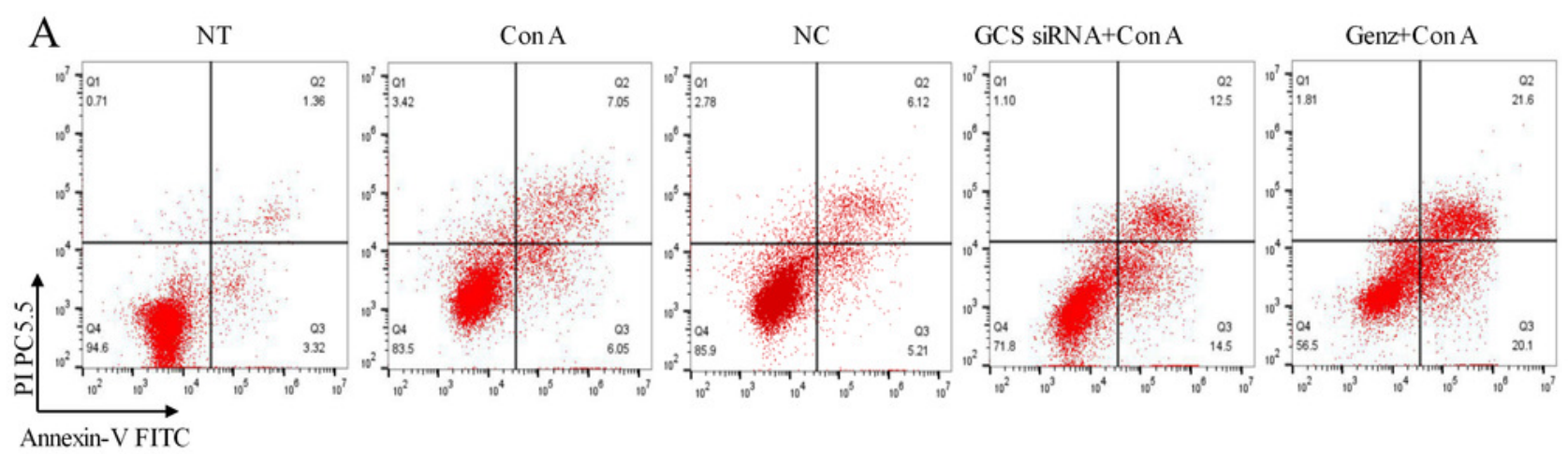

$\mathrm{B}$

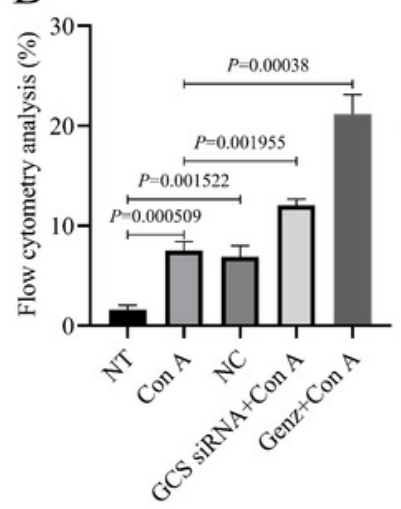

$\mathrm{C}$

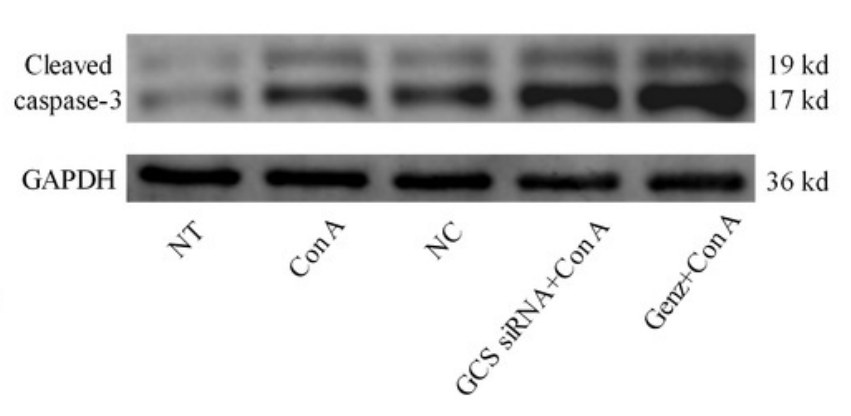

$\mathrm{D}$

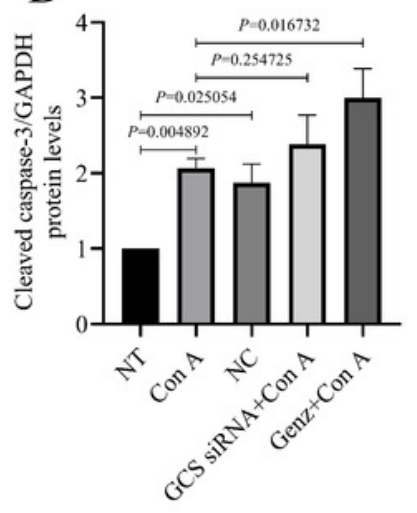


Figure 5

Serum IL-4, IL-6 and IL-10 levels.

(A) Serum IL-4 level. (B) Serum IL-6 level. (C) Serum IL-10 level.
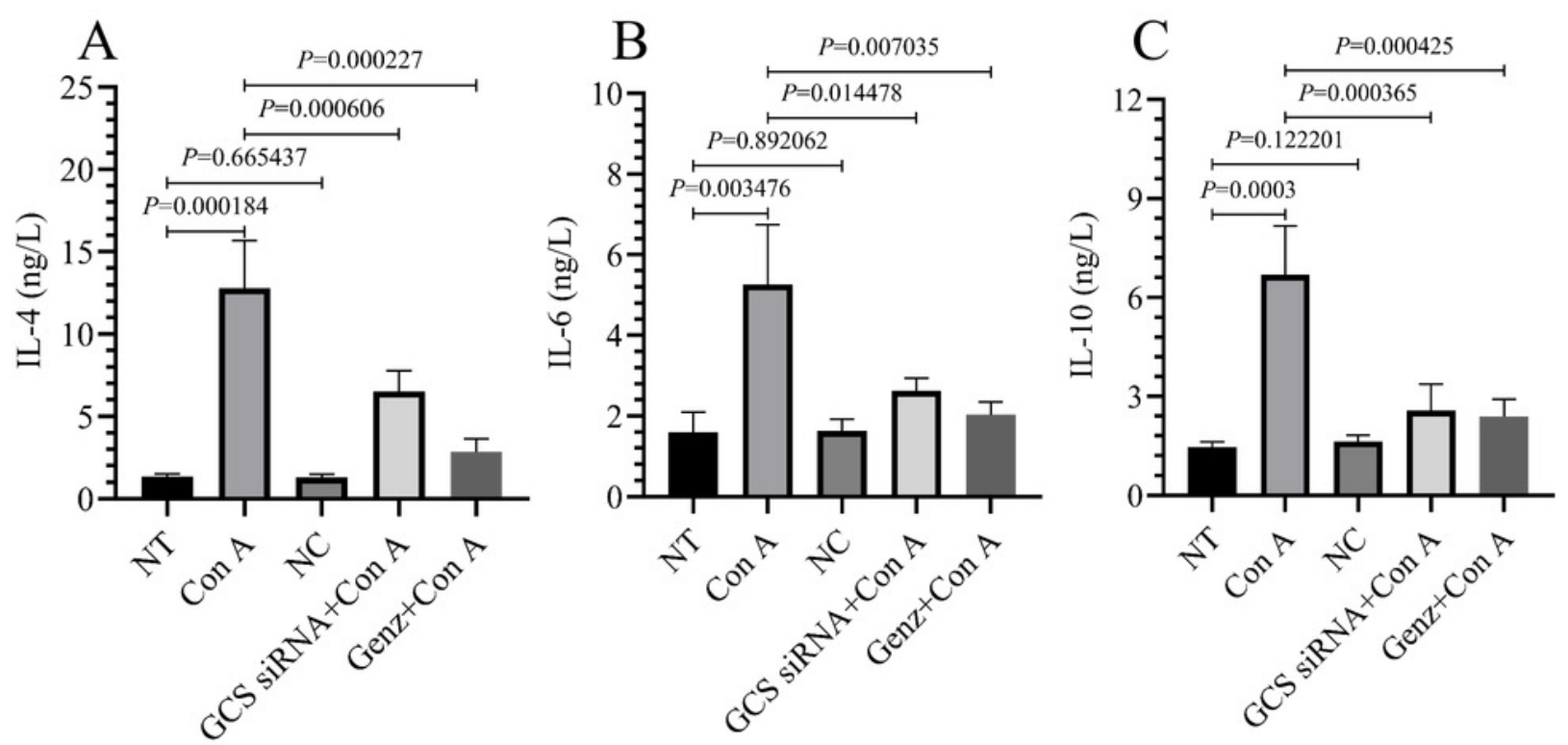
Figure 6

MMP-1 and TIMP-1 levels in liver.

(A) The relative expression level of MMP-1 mRNA measured by qRT-PCR method. (B) The relative expression level of TIMP-1 mRNA measured by qRT-PCR method. (C and D) MMP-1 and TIMP-1 proteins were analyzed by western blot. (E) Relative levels of MMP-1 protein corrected by GAPDH. (F) Relative levels of TIMP-1 protein corrected by GAPDH. 

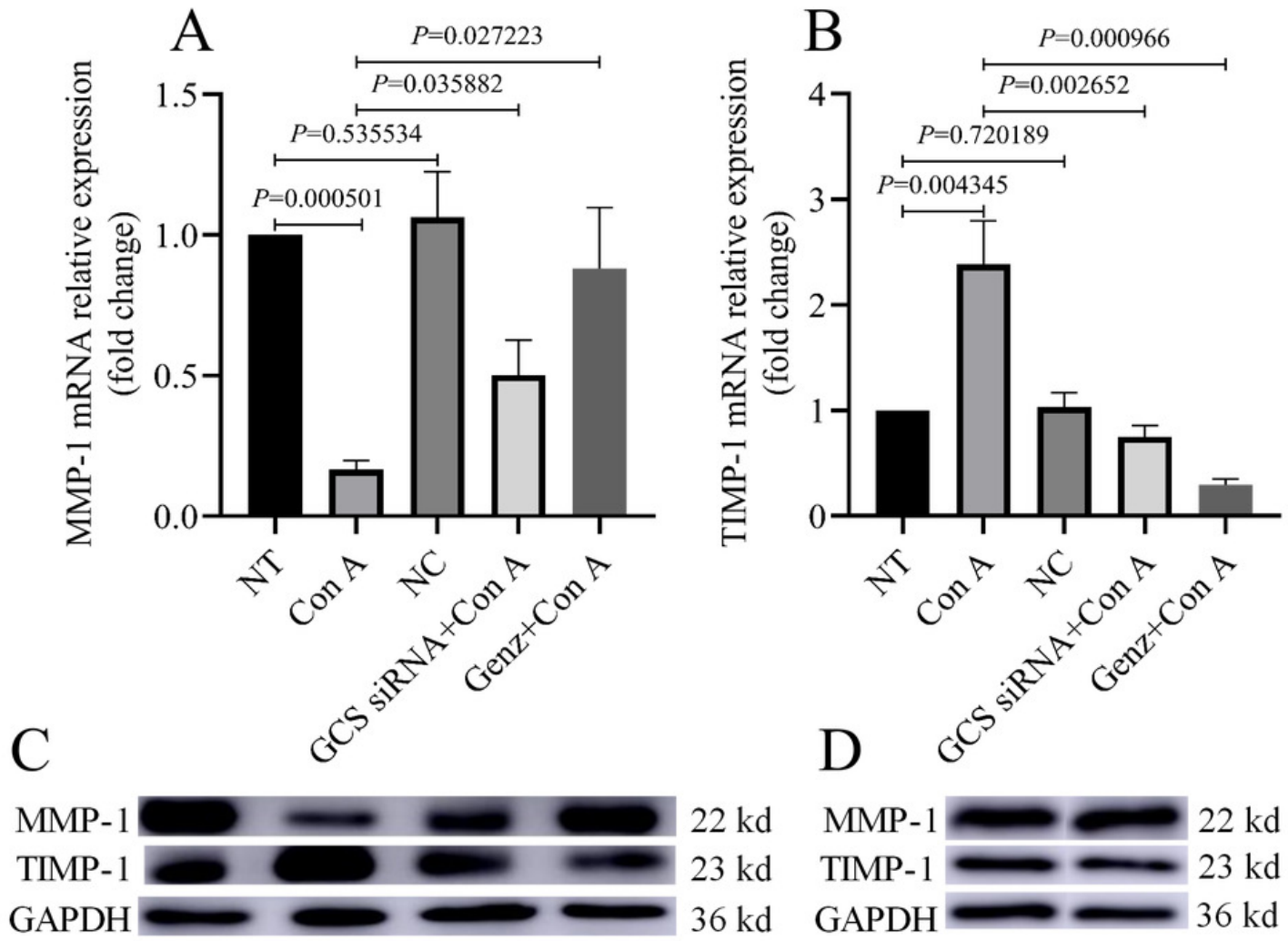

$22 \mathrm{kd}$ MMP-1 $22 \mathrm{kd}$

$23 \mathrm{kd}$ TIMP-1 $23 \mathrm{kd}$ $36 \mathrm{kd}$ GAPDH $36 \mathrm{kd}$

at $\cos \theta$

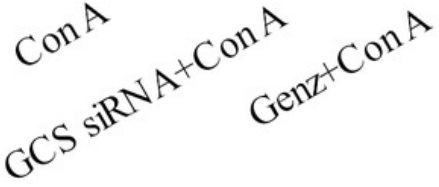
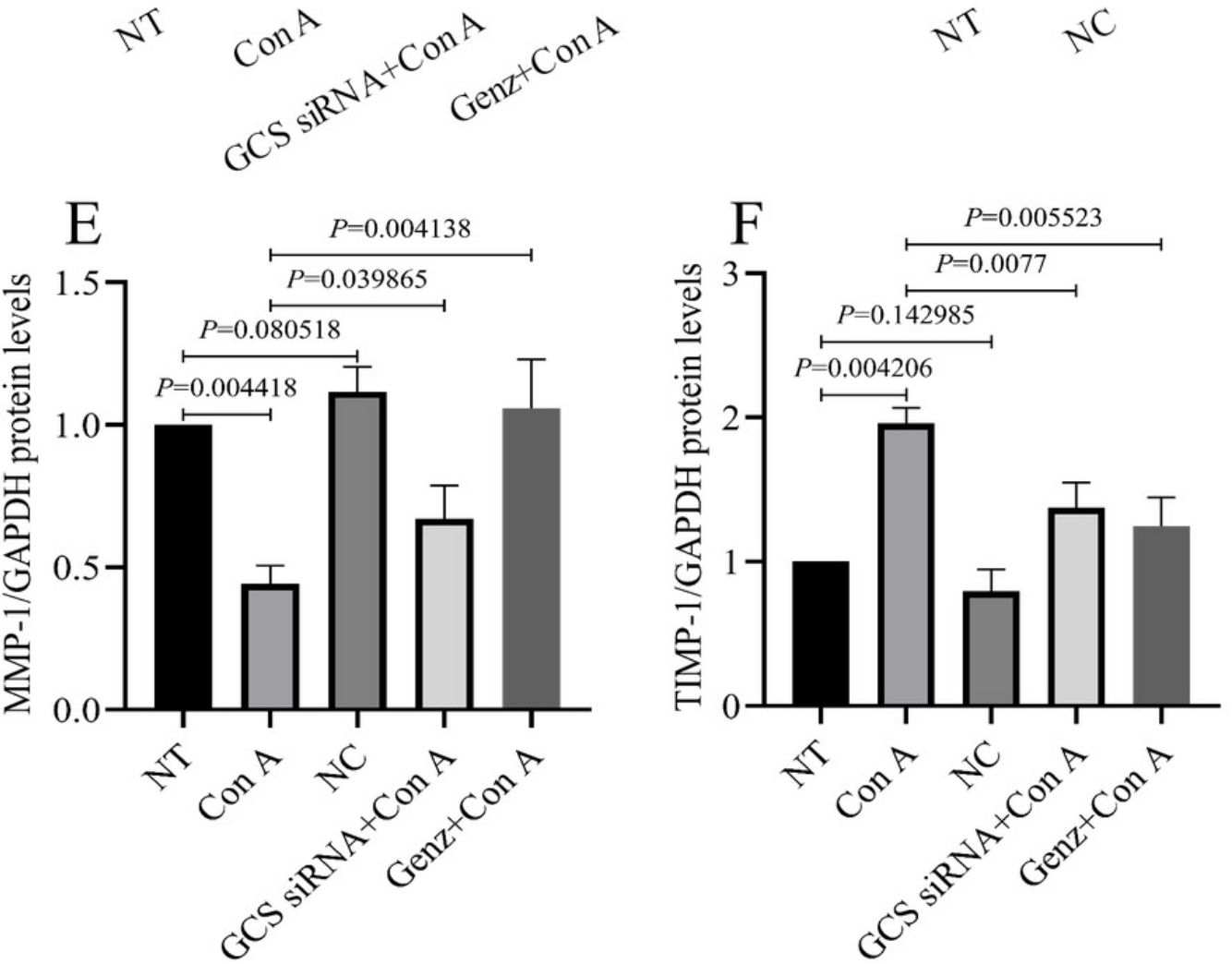